\title{
Intervenções do Terapeuta Psicanalítico no Processo Psicoterapêutico de uma Paciente com Transtorno de Personalidade Borderline
}

\author{
Liliam Simoni ${ }^{*}, 1$ \\ Orcid.org/0000-0003-3978-639X \\ Silvia Pereira da Cruz Benetti ${ }^{1}$ \\ Orcid.org/0000-0001-8557-2216 \\ Aline Alvares Bittencourt ${ }^{1}$ \\ Orcid.org/0000-0002-7121-145X
}

${ }^{1}$ Universidade do Vale do Rio dos Sinos, São Leopoldo, RS, Brasil

\section{Resumo}

Este estudo teve por objetivo identificar os tipos de intervenções de um terapeuta psicanalítico na psicoterapia de uma paciente com Transtorno de Personalidade Borderline, em um recorte do tratamento anterior à sua internação psiquiátrica. O procedimento adotado foi o Estudo de Caso Sistemático com delineamento quantitativo. Para análise dos dados, foram transcritas e analisadas doze sessões de psicoterapia, sendo as intervenções do terapeuta classificadas com base na Classificação Multidimensional de Intervenções Psicoterapêuticas Modificada (CMIP-M), que identifica tipos de intervenções em um material clínico. Duplas de juízes independentes e treinados na metodologia do instrumento avaliaram e classificaram cada uma das intervenções do terapeuta ao nível descritivo. Os resultados foram apresentados por meio de estatísticas descritivas de frequência e percentual e apontaram predominância das intervenções empáticas $(27,3 \%)$, exploratórias fechadas $(24,1 \%)$ e examinativas psicanalíticas $(15,1 \%)$ ao longo do processo investigado. Por um lado, os resultados mostraram semelhanças com estudos anteriores, indicando destaque às intervenções exploratórias fechadas no tratamento de transtornos de personalidade. Por outro lado, evidenciou-se diferenças em relação às investigações anteriores realizadas com pacientes diagnosticados com Transtorno de Personalidade Borderline, nas quais predominaram intervenções expressivas.

Palavras-chave: Psicoterapia psicanalítica, intervenção psicológica, Distúrbio de Personalidade Borderline.

\section{Interventions of the Psychoanalytic Therapist in the Psychotherapeutic Process of a Patient with Borderline Personality Disorder}

\begin{abstract}
This study aimed to identify the types of interventions of a psychoanalytic therapist in the psychotherapy of a patient with Borderline Personality Disorder, during a period of treatment prior to psychiatric

* Endereço para correspondência: Rua Carlos Mariani, 399, Bairro Rio Branco, Caxias do Sul, RS, Brasil 95097640. E-mail: liliamsimoni@terra.com.br, spcbenetti@gmail.com e alinealvares@hotmail.com
\end{abstract}


hospitalization. The Systematic Case Study procedure was adopted with a quantitative design. For the data analysis, twelve sessions of psychotherapy were transcribed and analyzed and the interventions of the therapist were classified based on the Modified Multidimensional Classification of Psychotherapeutic Interventions (CMIP-M), which identifies types of interventions in clinical material. Pairs of independent judges trained in the methodology of the instrument evaluated and classified each of the interventions of the therapist at the descriptive level. The results were presented by means of descriptive statistics of frequency and percentage and indicated predominance of empathic (27.3\%), closed exploratory (24.1\%) and psychoanalytical examination (15.1\%) interventions throughout the investigation process. The results showed similarities with previous studies, indicating the emphasis on closed exploratory interventions in the treatment of personality disorders. However, there were differences in relation to the previous investigations carried out with patients diagnosed with Borderline Personality Disorder, in which expressive interventions predominated.

Keywords: Psychoanalytic psychotherapy, psychological intervention, Borderline Personality Disorder.

\section{Intervenciones del Terapeuta Psicoanalítico en el Proceso Psicoterapéutico de uno Paciente con Trastorno de Personalidad Limítrofe}

\section{Resumen}

Este estudio ha tenido como objetivo identificar los tipos de intervención de un terapeuta en la psicoterapia psicoanalítica de un paciente con trastorno de personalidad limítrofe, en un recorte del tratamiento previo a su hospitalización psiquiátrica. El procedimiento adoptado fue el estudio de caso sistemático con un diseño cuantitativo. En el análisis de datos, se ha transcripto y analizado doce sesiones de psicoterapia, y las intervenciones del terapeuta clasificadas basadas en la Clasificación Multidimensional de Intervenciones Psicoterapéuticas, versión modificada (CMIP-M), que identifica los tipos de intervenciones en un material clínico. Un par de jueces independientes y entrenados en la metodología del instrumento de evaluación y clasificación de cada una de las intervenciones del terapeuta para el nivel descriptivo. Los resultados se presentaron mediante estadística descriptiva y el porcentaje de frecuencia y revelaron predominio de intervenciones empáticas $(27,3 \%)$, exploratorios cerrada $(24,1 \%)$ y examinativas psicoanalíticas $(15,1 \%)$ en el proceso de investigación. Por un lado, los resultados sugirieron similitudes con estudios previos que indican énfasis en intervenciones cerradas exploratorias en el tratamiento de trastornos de la personalidad. Por otro lado, mostró diferencias en relación a las investigaciones llevadas a cabo con los pacientes diagnosticados con el trastorno de personalidad limítrofe, en la que predominaban las intervenciones expresivas.

Palabras clave: Psicoterapia psicoanalítica, intervención psicológica, trastorno de personalidad limítrofe.

As pesquisas de processo em psicoterapia constituem um campo fértil para investigação e um desafio para pesquisadores e clínicos. A prática clínica baseada em evidências científicas, por meio da análise dos processos e dos resultados psicoterápicos, aprimora o trabalho clínico com pacientes e permite uma melhor compreensão sobre as psicoterapias (Brum et al., 2012; Peuker, Habigzang, Koller, \& Araujo, 2009).
Uma área que tem merecido significativa atenção em termos de pesquisa, devido ao aumento da demanda clínica atual, é a referente à investigação do processo psicoterápico dirigido a pacientes graves, principalmente com sintomas associados ao diagnóstico de Transtorno de Personalidade Borderline (Eizirik \& Fonagy, 2009; Fonagy \& Bateman, 2007; Jordão \& Ramires, 2010). Esses pacientes, em função de atitudes de 
impulsividade e agressividade, podem apresentar dificuldades de adesão aos tratamentos (Tanesi, Yazigi, Fiore, \& Pitta, 2007). A psicoterapia, embora difícil de ser executada e associada a altas taxas de evasão nesses casos, é considerada adequada para o tratamento do Transtorno de Personalidade Borderline (Madeddu, Aquaro, \& Petri, 2012).

Para o aprimoramento das psicoterapias em geral, um dos aspectos extensamente investigados em distintos modelos teóricos refere-se ao estudo dos mecanismos de ação terapêutica, no qual se destaca a contribuição do terapeuta, do paciente e da interação em relação a diversos aspectos que afetam as psicoterapias (Peuker et al., 2009). Dentre os mecanismos de ação terapêutica, as variáveis do terapeuta constituem um dos elementos relevantes de investigação (Goodman, Anderson, \& Diener, 2014), pois podem influenciar o processo de psicoterapia e seus resultados (Cordioli \& Giglio, 2008).

Alguns autores entendem que as características pessoais do terapeuta possuem significado sobre os tratamentos (Heinomen, Knekt, Jääskeläinen, \& Lindfors, 2014; Lambert, 2013). Lambert (2013) propõe que dentre as variáveis do terapeuta que podem interferir nas psicoterapias estão sexo, idade, etnia, experiência, personalidade, bem-estar emocional, valores, crenças, orientação teórica, dentre outras. Outro aspecto considerado relevante dentre as variáveis do terapeuta refere-se às características das intervenções realizadas ao longo do processo psicoterápico (Goodman et al., 2014). Esse foco de pesquisa permite conhecer os tipos de intervenções associadas à melhoria ou à alteração do processo, identificando, assim, os chamados "ingredientes ativos" da mudança terapêutica (Banon et al., 2013; Roussos, Waizmann, \& Etchebarne, 2010).

Verifica-se que os terapeutas exercem grande impacto nos resultados dos tratamentos (Owen, Drinane, Idigo, \& Valentine, 2015). Apesar dos avanços da literatura internacional e das pesquisas em psicoterapia, estudos embasados no modelo psicanalítico ainda são escassos. Existem poucas pesquisas brasileiras que buscaram investigar as variáveis dos terapeutas en- volvidas nos tratamentos (Quadros \& Yoshida, 2012).

Ao longo dos anos, é possível observar que ocorreram diferentes ênfases na compreensão da ação terapêutica na abordagem psicanalítica. Ainda que a interpretação, o insight e os aspectos da interação terapêutica entre analista e paciente constituem métodos centrais na ação terapêutica, esses elementos não são identificados como únicos no processo terapêutico. Atualmente, a maioria dos terapeutas psicanalíticos reconhece que há múltiplos modos de ação terapêutica que variam de paciente para paciente (Clarkin, Fonagy, \& Gabbard, 2013; Gabbard, 2005; Gabbard \& Westen, 2003). Nesse sentido, ocorreram modificações no manejo da técnica psicoterápica de determinados pacientes, em especial no tratamento de portadores de patologias graves (Pasini \& Dametto, 2010). Ao corroborar com essa posição, Quagliatto e Santos (2004) destacaram modificações no manejo da técnica psicoterápica de determinados pacientes, principalmente com aqueles caracterizados como portadores de psicopatologias graves: psicoses, transtorno borderline, transtorno narcisista, psicopatias, entre outros.

Com relação ao aspecto técnico das intervenções do terapeuta, verifica-se que as técnicas básicas da psicoterapia psicanalítica com pacientes com TPB são as mesmas da psicanálise: interpretação, análise da transferência e neutralidade técnica. Entretanto, essas técnicas são usadas de forma diferente pelo terapeuta (Kernberg, 2003; Kernberg, Selzer, Hoenigsberg, Carr, \& Appelbaum, 1991).

Kernberg (2003) refere que a clarificação, confrontação e interpretação são os principais aspectos da técnica interpretativa utilizada na psicoterapia psicanalítica. Com pacientes com psicopatologias sérias, a clarificação e a confrontação ocupam um espaço mais amplo do que a interpretação e as interpretações dos significados inconscientes no "aqui e agora" tem maior espaço do que aquelas no "lá e então". Somente em estágios mais avançados do tratamento são introduzidas interpretações além do "aqui e agora", de forma a relacionar o comportamento passado com o presente. Além disso, as interpre- 
tações são mais eficazes quando apresentadas a um paciente preparado emocionalmente. Assim, o uso sequencial de clarificação e de confrontação abrem caminho para a interpretação propriamente dita.

Outra técnica básica da psicanálise, segundo Kernberg (2003), é a análise da transferência, que é o foco principal da interpretação na psicanálise clássica. Ela é modificada com pacientes com graves distúrbios de personalidade, ao considerar qualquer interpretação transferencial no contexto do que está ocorrendo na vida atual do paciente (Kernberg et al., 1991). A transferência negativa e positiva desses pacientes deve ser trabalhada apenas no "aqui e agora" sem que se tente atingir reconstruções genéticas completas, pois a falta de diferenciação e individualização dos objetos interfere na capacidade para diferenciar os relacionamentos objetais presentes e passados (Kernberg, 1995).

A neutralidade técnica do terapeuta também é considerada um elemento essencial do método psicanalítico, a qual é apontada como um estado ideal de trabalho. Contudo, em pacientes com sérias patologias de personalidade, a necessidade de determinar limites impõe modificações temporárias na técnica para permitir controlar atuações que ameaçam a vida do paciente ou o tratamento (Kernberg, 2003).

Apesar da ampliação no conhecimento acerca do trabalho clínico com pacientes graves, ainda hoje estudos sobre o processo psicoterápico em relação às intervenções do terapeuta são necessários (Lambert, 2013; Quadros \& Yoshida, 2012). Conforme Banon et al. (2013), as pesquisas de processo em psicoterapia requerem uma base descritiva das características da técnica do terapeuta. Assim, identificar intervenções do terapeuta facilita os estudos de processo ao elucidar conexões entre tipos de intervenções e seus efeitos no processo de mudança.

No tocante às pesquisas, grande parte dos estudos investiga as intervenções dos terapeutas frente às diferentes abordagens teóricas. Uma pesquisa realizada por Banon et al. (2013), utilizando como instrumento a Psychodynamic Intervention Rating Scale (PIRS), teve por objetivo avaliar a frequência e a profundidade das inter- venções do terapeuta nas psicoterapias psicodinâmica, psicanálise e cognitiva comportamental. Dentre os diversos resultados, foi mencionado que as proporções de interpretações aumentaram ao longo do tempo, bem como sua profundidade. As interpretações de defesa foram mais comuns do que a interpretação de transferência, que foram mais prevalentes na psicanálise.

Alguns autores se dedicaram a analisar as intervenções do terapeuta no que se refere ao marco teórico psicanalítico e cognitivo comportamental. Um estudo realizado por Waizmann, Jurkowski e Roussos (2006), com o objetivo de verificar a modalidade de intervenções dos terapeutas psicanalíticos e cognitivos, buscou indagar acerca dos tipos de intervenções que utilizam em termos de intervenções específicas e não específicas. Entre os resultados obtidos, os autores observaram que os terapeutas utilizavam intervenções específicas de outro marco teórico de referência, porém se mantinham fiéis a seu próprio marco em relação às intervenções que mais utilizavam.

Por outro lado, um estudo posterior realizado por Roussos et al. (2010), utilizando como instrumento a Classificação Multidimensional de Intervenções Psicoterapêuticas Modificada (CMIP-M), encontrou resultados contraditórios. O CMIP apresenta uma metodologia para a construção de um esquema classificatório das intervenções psicanalíticas e cognitivo comportamentais e tem como objetivo identificar tipos de intervenções em um material clínico. A CMIP-M considera quatro níveis de análise das intervenções, sendo elas: (1) estratégico, (2) descritivo, (3) de conteúdo e (4) espaço-temporal (Roussos, Etchebarne, \& Waizmann, 2005).

Os autores do estudo utilizaram o nível descritivo do instrumento, que avalia os distintos tipos e formas de intervenções, para examinar as intervenções utilizadas em dois tratamentos psicoterapêuticos, um cognitivo e um de orientação psicanalítica, ambos com diagnóstico de transtorno de personalidade. Esse nível descritivo inclui os seguintes tipos de intervenções: exploratórias, empáticas, explicativas, indicativas, examinativas, com terceiras partes (pessoas externas a díade) e não classificável. Além disso, 
observaram se as intervenções dos terapeutas foram diretamente associadas com a sua base teórica, e qual a proporção de intervenções comuns, inespecíficas e específicas. Nesse último caso, intervenções comuns são as utilizadas por ambos os marcos teóricos, intervenções inespecíficas são as utilizadas por outro marco teórico que não seja a do terapeuta tratante e, específicas, são aquelas próprias da linha teórica do psicoterapeuta (Roussos et al., 2005). Os resultados mostraram que ambos os tratamentos foram altamente impregnados por intervenções inespecíficas. Apenas uma média de $17 \%$ das intervenções no tratamento psicanalítico e uma média de $16 \%$ no tratamento cognitivo foram específicas dos quadros teóricos. Diferenças encontradas nas proporções de intervenções específicas e comuns podem ser justificadas a partir do pressuposto de que ambos os tipos de intervenções interagem e se complementam. Assim, as intervenções comuns podem estar abrindo o caminho para as específicas, isto é, a sua função na psicoterapia pode ser a de gerar o contexto e o momento certo para as intervenções específicas (Roussos et al., 2010).

Nota-se que os estudos focalizam a identificação das intervenções terapêuticas de acordo com os modelos teóricos, mas poucos investigam as intervenções psicoterapêuticas frente aos distintos quadros clínicos. Em relação a pacientes graves, foram observados limitados investimentos científicos que se dedicaram ao estudo das intervenções psicoterapêuticas. Koenigsberg, Kernberg, Appelbaum e Smith (1993) desenvolveram um método para analisar intervenções do terapeuta na psicoterapia com pacientes Borderline, o qual monitorava o uso da técnica expressiva e de apoio nas sessões. Dentre os resultados encontrados, destacaram que a porcentagem total de intervenções expressivas (esclarecimentos, confrontação e interpretação) variou de $68 \%$ a $98 \%$ entre as 12 sessões analisadas, com exceção de um caso isolado (43\%). Os índices de esclarecimento, confrontação e interpretação foram uniformes, sem discrepâncias claras. Essas sessões foram conduzidas por dez terapeutas que variaram em tempo de experiência e formação em psicanálise, bem como houve uma mistura de sessões precoces e tardias.
Alguns autores colocam que conforme a sintomatologia do paciente no transcorrer do atendimento, o terapeuta modifica suas intervenções. Nesse caso, Goodman et al. (2014) verificaram que terapeutas de orientação psicanalítica, frente a pacientes gravemente perturbados, requerem uma flexibilidade temporária da técnica e adotam intervenções mais estruturadas, que servem para a estabilização do tratamento.

Observa-se que as psicoterapias têm se mostrado eficazes por meio de diferentes abordagens e em diferentes transtornos, sendo que tanto fatores comuns como fatores específicos têm sido investigados e relacionados ao sucesso terapêutico (Brum et al., 2012; Isolan, Pheula, \& Cordioli, 2008). Todavia, percebe-se a necessidade de um maior número de estudos dirigidos para a identificação das intervenções do terapeuta de forma a contribuir para o avanço do conhecimento em relação à ação terapêutica com base em distintas abordagens teóricas e quadros clínicos.

Assim, esse estudo teve por objetivo identificar os tipos de intervenções utilizadas por um terapeuta de orientação psicanalítica durante o processo de psicoterapia de uma paciente com Transtorno de Personalidade Borderline, em um recorte do tratamento anterior à sua internação psiquiátrica, Foi verificado a proporção de cada tipo de intervenção ao longo do processo e, posteriormente, nas três etapas divididas do tratamento. Também foi investigada a proporção de intervenções comuns e intervenções específicas psicanalíticas ao longo do tratamento analisado.

\section{Método}

\section{Participantes}

O caso em estudo é de uma paciente de 18 anos, estudante universitária, que buscou tratamento em uma clínica psiquiátrica de Porto Alegre, município localizado no estado do Rio Grande do Sul, Brasil. A jovem residia com a mãe, tendo o pai falecido durante sua infância. $\mathrm{Na}$ entrevista de triagem, a paciente apresentava crises de choro e relatou várias tentativas de cortar-se. Disse ter realizado psicoterapia em diversos momentos de sua vida, com um histórico de abandonos precoces dos tratamentos. 
Passado um período após a realização da triagem, a paciente foi encaminhada para psicoterapia individual de orientação psicanalítica com frequência de duas vezes por semana e também para avaliação psiquiátrica. $\mathrm{O}$ diagnóstico realizado pelo psiquiatra durante a etapa inicial do tratamento psicoterápico foi de Transtorno de Personalidade Borderline. A paciente iniciou tratamento medicamentoso antidepressivo e, durante o período do processo psicoterápico investigado, não houve mudança de dosagem ou medicação.

A terapeuta possui formação em psicoterapia de orientação psicanalítica e tem como referência autores psicodinâmicos. Sua prática é baseada na tríplice formação: seminários teóricos, supervisões e tratamento pessoal. Possui dez anos de experiência em atendimento psicoterápico. Cabe ressaltar que a pesquisadora não é a terapeuta do caso em estudo.

\section{Instrumento}

Nesta pesquisa utilizou-se como instrumento a Classificação Multidimensional de Intervenções Psicoterapêuticas Modificada (CMIP-M), desenvolvida por Roussos et al. (2005). O instrumento foi traduzido do espanhol para o português por um profissional proficiente em espanhol com formação em Psicologia e posteriormente foi realizada a retroversão por outra profissional bilíngue.

A CMIP-M apresenta uma metodologia para a construção de um esquema classificatório das intervenções psicoterapêuticas psicanalíticas e cognitivo-comportamentais. O objetivo da mesma foi construir um esquema de compreensão para identificar tipos de intervenções em um material clínico (Roussos et al., 2005).

Segundo os mesmos autores, a CMIP-M considera quatro níveis de análise das intervenções, sendo eles: (1) estratégico, (2) descritivo, (3) de conteúdo e (4) espaço-temporal. Os níveis apresentam interações entre si, mas podem ser avaliados de forma independente, pois representam diferentes aspectos de um mesmo evento. No presente estudo, foram avaliadas as intervenções de um terapeuta psicanalítico considerando apenas o nível descritivo.
O nível descritivo avalia as verbalizações realizadas pelo terapeuta e que são intencionalmente dirigidas para o paciente. É o estudo exclusivo dos distintos tipos e formas de intervenções. Para classificar o nível descritivo das intervenções sempre se deve respeitar a conceituação do marco teórico do terapeuta tratante psicanalítico ou cognitivo-comportamental, uma vez que a CMIP-M apresenta subtipos de grupos de intervenções comuns a ambos os marcos teóricos, subtipos psicanalíticos, subtipos cognitivos e subtipos comportamentais (Roussos et al., 2005).

No que se refere às intervenções a nível descritivo específicas ao marco teórico psicanalítico e comuns a ambos os marcos, considera-se sete grupos de intervenções. São eles: (1) Exploratórias (demanda informação aberta, demanda informação fechada, aplicação de escalas e questionários); (2) Empáticas (antecipação, repetição, síntese, ordem fática, aquiescência, apoio); (3) Explicativas (explicação geral, explicação psicológica, meta intervenção, interpretação, interpretação transferencial); (4) Indicativas (sugestão ou conselho, definição de limites, instruções de enquadre, associação livre); (5) Examinativas (recapitulação, sinalização, confrontação); (6) Com terceiras partes (pessoas externas a díade) e (7) Não classificável. Três desses grupos comportam intervenções específicas psicanalíticas, sendo que dentro do grupo das intervenções explicativas, a interpretação e a interpretação transferencial são específicas psicanalíticas, assim como dentro do grupo das indicativas, a associação livre e dentro do grupo das examinativas, a sinalização e a confrontação (Roussos et al., 2005). Ressalta-se que a sinalização é entendida como intervenções de esclarecimento (clarificação).

A CMIP-M, desenvolvida com base na investigação teórica e na análise qualitativa de sessões clínicas gravadas em áudio (Roussos et al., 2010), já foi utilizada em outros estudos com o objetivo de analisar as intervenções psicoterapêuticas. Autores como Roussos et al. (2010) e Waizmann et al. (2006) fizeram uso da CMIP-M em seus estudos e não mencionaram nenhuma limitação referente ao instrumento. 


\section{Procedimentos de Coleta de Dados}

Todas as sessões de psicoterapia foram gravadas em vídeo. Para este estudo foram transcritas e analisadas 12 sessões de um total de 34 que antecederam a internação psiquiátrica da paciente. Esse período corresponde ao primeiro ano de tratamento da paciente, e inclui as seguintes sessões: $1,4,7,10,13,16,19,22,25,28,31$ e 34 . A escolha das sessões se deu de forma aleatória, considerando 1 a cada 3 sessões, procurando-se dessa forma, acompanhar a evolução do caso sem perder sua especificidade.

\section{Procedimentos de Análise de Dados}

Duplas de juízes independentes e treinados na metodologia de avaliação da CMIP-M assistiram as gravações das sessões e, em posse da transcrição da mesma e do manual de instrução do instrumento, avaliaram as sessões de psicoterapia de forma a classificar cada uma das intervenções do terapeuta ao nível descritivo. Para verificar a confiabilidade das classificações, realizou-se supervisão de uma sessão com o autor do instrumento e foi calculado o coeficiente de variabilidade entre juízes - Kappa, atingindo-se concordância superior a 0,7 em todas as sessões investigadas. Participaram das classificações três juízes devidamente treinados quanto aos procedimentos de classificação, sendo que se efetuou revezamento na formação das duplas.

Os tipos de intervenções foram avaliados de forma quantitativa, sendo que todas as análises foram realizadas através do Statistical $\mathrm{Pa}$ ckage for Social Sciences (SPSS) versão 20.0. Os resultados estão apresentados por meio de estatísticas descritivas de frequência e percentual.

Além disso, foram identificadas três etapas do tratamento, sendo elas: (1) inicial, (2) intermediária e (3) anterior à internação. As primeiras quatro sessões analisadas $(1,4,7$ e 10) correspondem à etapa inicial do tratamento, as quatro sessões seguintes $(13,16,19$ e 22$)$ correspondem à segunda etapa ou etapa intermediária e as últimas sessões investigadas $(25,28,31 \mathrm{e}$ 34) correspondem à terceira etapa do tratamento, a qual antecede a internação psiquiátrica da paciente.
Por último, as intervenções foram analisadas quanto a sua especificidade, verificando-se a proporção de intervenções específicas psicanalíticas e comuns a ambos os marcos teóricos. Foram denominadas como "intervenções específicas psicanalíticas" as verbalizações realizadas pelo terapeuta que são específicas do marco teórico psicanalítico (Waizmann, Etchebarne, \& Roussos, 2004). De acordo com a denominação dos mesmos autores, foram consideradas como "intervenções comuns" aquelas verbalizações do terapeuta que não formam parte explícita de sua orientação teórica, mas que são utilizadas em ambas as formas de psicoterapia (Psicanalítica e Cognitivo-Comportamental).

\section{Procedimentos Éticos}

A paciente e a terapeuta leram e assinaram o Termo de Consentimento Livre e Esclarecido. Todos os cuidados éticos da Resolução $n$. 466/2012 do Conselho Nacional de Saúde foram respeitados (2012), tendo sido concordada a gravação em vídeo das sessões pela terapeuta e paciente.

\section{Resultados}

Foram consideradas todas as intervenções verbais do terapeuta durante cada uma das 12 sessões e classificadas de acordo com o nível descritivo da CMIP-M. Os resultados são apresentados na Figura 1, na qual se encontram as distribuições das frequências de cada tipo de intervenção terapêutica utilizada ao longo do processo investigado. Nota-se que predominaram as intervenções empáticas com 27,3\% e as exploratórias fechadas, que atingiram um percentual de 24,1\%. Essas intervenções vieram seguidas das examinativas psicanalíticas $(15,1 \%)$, exploratórias abertas $(13,1 \%)$ e não classificáveis $(12,1 \%)$. As frequências mais baixas ocorreram nas intervenções explicativas psicanalíticas $(0,2 \%)$, examinativas comuns $(0,7 \%)$, indicativas comuns $(2,5 \%)$ e explicativas comuns $(5,1 \%)$. As intervenções indicativas psicanalíticas e com terceiras partes não ocorreram no processo investigado. 


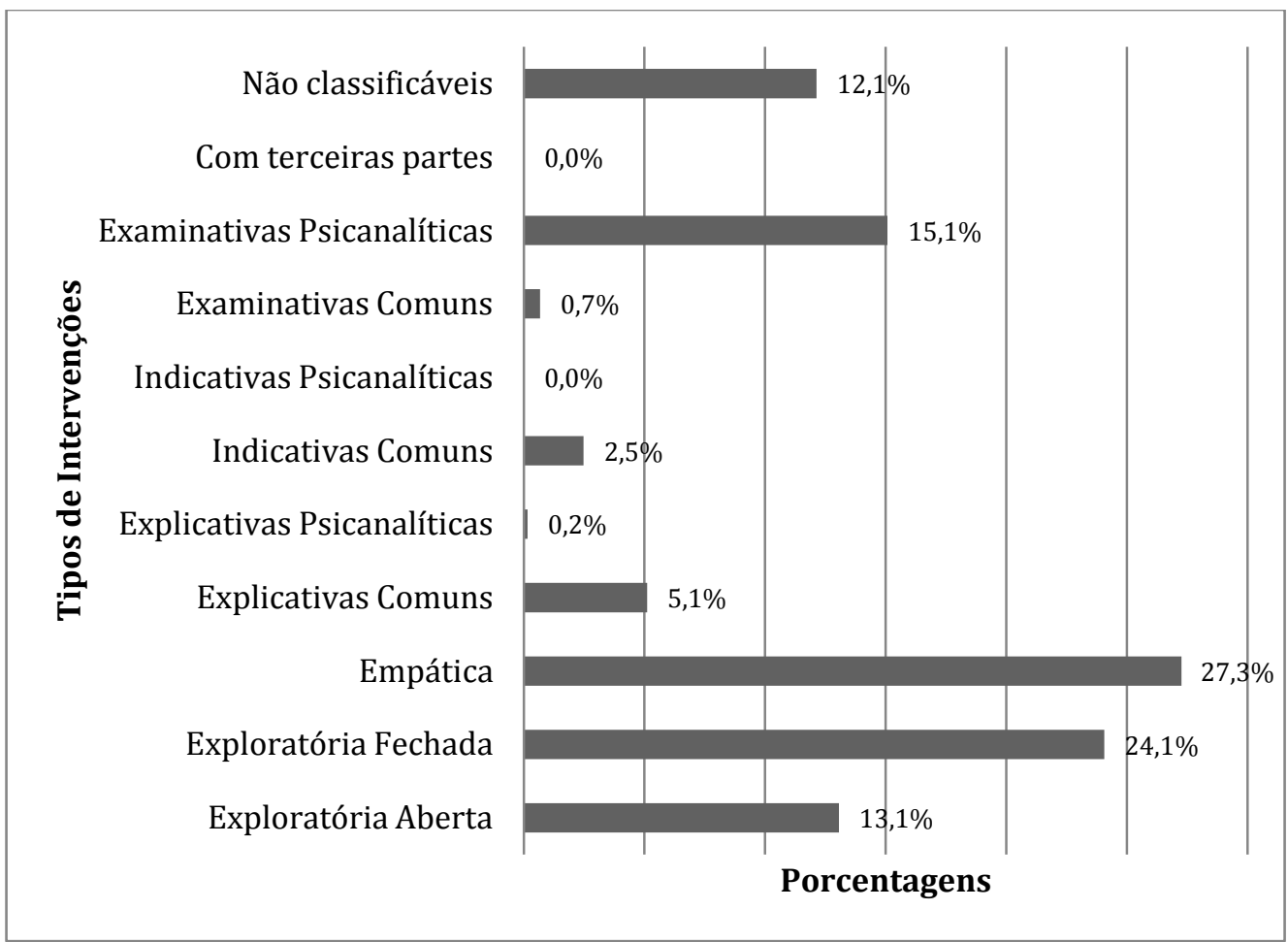

Figura 1. Proporção de cada tipo de intervenção ao longo do processo investigado.

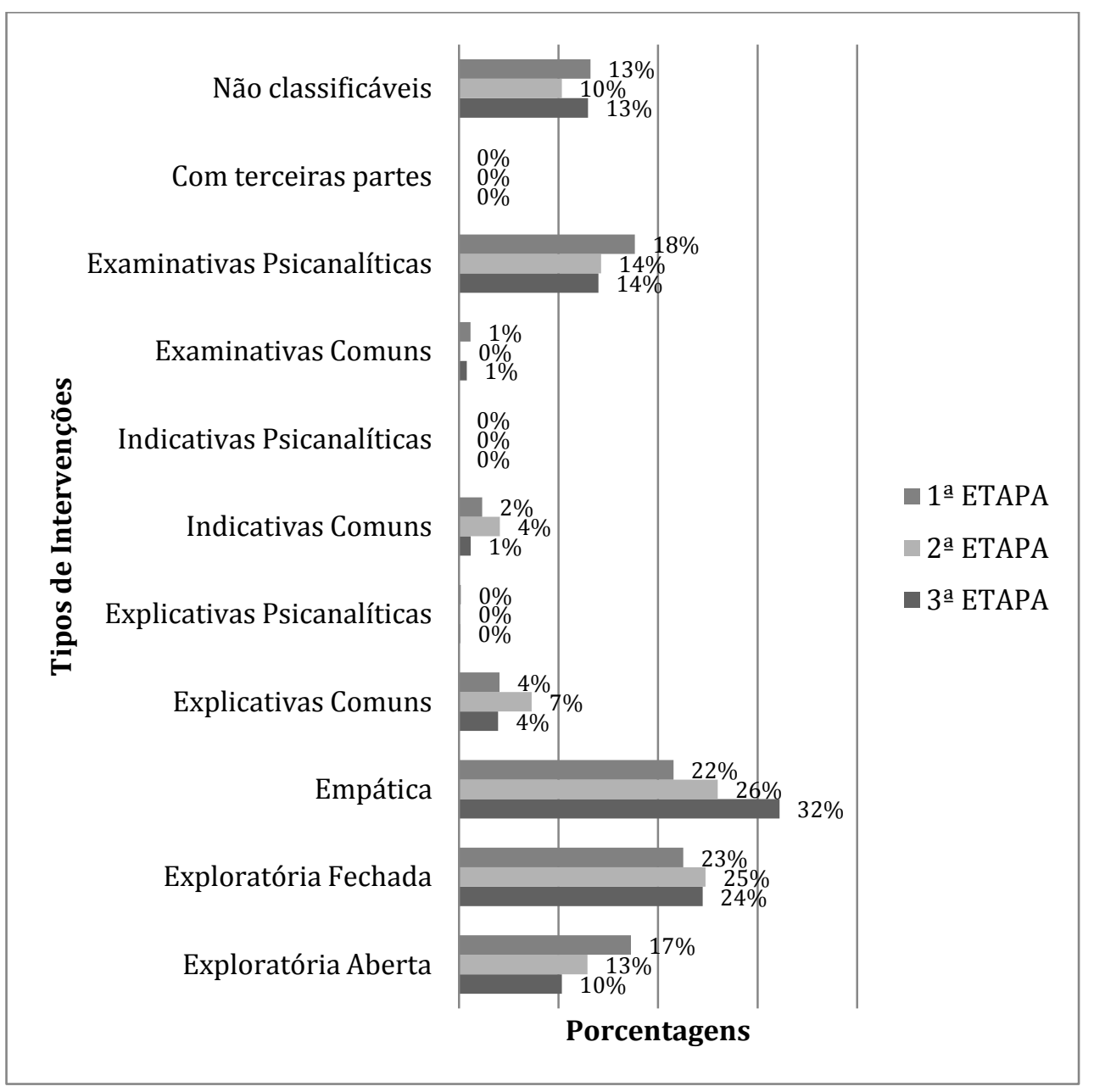

Figura 2. Intervenções predominantes por etapa do tratamento. 
Neste estudo também foram identificadas três etapas as quais foram exploradas em relação às intervenções predominantes. Na Figura 2, observa-se que predominaram em todas as etapas as intervenções empáticas e exploratórias fechadas. Assim, na primeira etapa do processo psicoterápico, as intervenções exploratórias fechadas $(23 \%)$ foram seguidas das empáticas (22\%), na segunda etapa as empáticas (26\%) foram seguidas das exploratórias fechadas $(25 \%) \mathrm{e}$ na terceira etapa, ocorreu à frequência máxima das intervenções empáticas (32\%) que foram seguidas das exploratórias fechadas (24\%).

A análise específica dessas proporções indica que a oscilação máxima de cada tipo de intervenção por etapa foi de $10 \%$. Essa oscilação se deu entre a primeira e a terceira etapa em relação às intervenções empáticas. Os demais tipos de intervenção mantiveram proporções equivalentes entre as três etapas do processo investigado. Ao observar o trabalho do terapeuta ao longo do tratamento em relação às intervenções empáticas, verifica-se que o aumento desse tipo de intervenção na terceira etapa correspondeu a um trabalho mais focalizado na situação do paciente em crise, uma vez que essas sessões correspondem ao período anterior à internação.

Por último, ao analisar a proporção de intervenções comuns e intervenções específicas de base psicanalíticas ao longo do processo psicoterápico investigado, é possível constatar que $15 \%$ das intervenções foram específicas à orientação psicanalítica, enquanto que $85 \%$ foram intervenções comuns. Assim, observa-se que as intervenções se caracterizaram, em sua maioria, como comuns, ou seja, que são utilizadas em tratamentos psicanalíticos e cognitivo-comportamentais.

\section{Discussão}

Este estudo dirigiu-se à investigação da ação terapêutica no modelo de orientação psicanalítico de psicoterapia dirigido a pacientes graves, considerando especificamente a contribuição do terapeuta no processo. Teve por objetivo identificar os tipos de intervenções de um terapeuta psicanalítico durante o processo de psicoterapia de um paciente com Transtorno de Personalida- de Borderline. A avaliação da proporção de cada tipo de intervenção ao longo do processo investigado revela que predominaram as intervenções empáticas e as exploratórias fechadas. O terceiro tipo de intervenção mais utilizado pela terapeuta do caso foram as examinativas psicanalíticas.

Os tipos de intervenções empregadas pela terapeuta em maiores proporções parecem condizentes com o modelo de funcionamento de pacientes com TPB. Esses se caracterizam por sérios comportamentos de autoagressão e atitudes suicidas (Madeddu et al., 2012), frequentes atuações, tendência a impulsividade e agressividade (Bleichmar, 1992; Hallquist \& Pilkonis, 2012) e dificuldades de adesão ao tratamento (Tanesi et al., 2007).

Ao considerar essas características, a terapeuta utilizou-se com maior frequência das intervenções empáticas, uma vez que essa postura terapêutica contribui para adaptação do paciente e estabelecimento do vínculo. Alguns autores referem que estabelecer uma aliança terapêutica com pacientes graves é muito importante para o manejo do tratamento, pois tais pacientes apresentam características peculiares que influenciam na sua adesão e também na decisão do uso da técnica pelo psicoterapeuta (Owen \& Hilsenroth, 2011). Observa-se que a terapeuta estava focalizada em estabelecer uma aliança terapêutica, já que a qualidade da mesma é um forte preditor de resultados (Elliot, 2012).

A atitude empática da terapeuta, conforme Ablon e Jones (1998), está de acordo com as técnicas terapêuticas da abordagem psicanalítica. Os autores descrevem que o modelo de base psicanalítico se destaca por uma posição do terapeuta neutra, empática e sensível aos sentimentos do paciente.

O segundo tipo de intervenção mais utilizado foi às exploratórias fechadas, quando a terapeuta realiza pedidos de informações, introduzindo tópicos de discussão. Em geral, o uso de intervenções interrogativas, caracterizam uma postura mais ativa e diretiva do terapeuta. Geralmente, é mais utilizada no modelo cognitivo de tratamento. Entretanto, frente à pacientes com psicopatologias sérias, terapeutas de orientação psicanalíticos flexibilizam a técnica ao visar à 
estabilização do tratamento (Goodman, Edwards, \& Chung, 2013).

Nesse sentido, Goodman et al. (2013) analisaram o processo psicoterápico de cinco pacientes com TPB e associaram a diferentes modelos terapêuticos. Nessa pesquisa, a postura empática correlacionou-se com redução sintomática. Da mesma forma, o terapeuta exercer ativamente o controle da interação, questionando e solicitando mais informações, associou-se à redução sintomática. Segundo os pesquisadores, a utilização dessas estratégias por terapeutas psicodinâmicos indica o esforço de manter a estabilidade de pacientes graves e a aliança terapêutica, quando em face à intensificação sintomática.

Dessa forma, pode-se relacionar a prevalência das intervenções empáticas e exploratórias fechadas no processo investigado como tendo relação à sintomatologia da paciente e ao diagnóstico do caso. Em contrapartida, a preponderância de tais intervenções, também pode estar associada a particularidades da terapeuta. Portanto, é possível questionar o quanto os resultados referem-se ao diagnóstico Borderline e sua sintomatologia ou a terapeuta, uma vez que autores como Fernández-Alvarez, Gomez e Garcia (2015) e Lambert (2013) verificaram que o psicoterapeuta pode influenciar no desenvolvimento e nos resultados do processo psicoterápico.

Frente a essas questões, no tocante as pesquisas já realizadas, se observam resultados distintos. Por um lado, em similaridade com o presente estudo, Roussos et al. (2010), utilizando como instrumento a CMIP-M, analisaram as intervenções em um tratamento psicanalítico com diagnóstico de transtorno de personalidade não especificado e verificaram uma maior proporção de intervenções do tipo interrogações fechadas. Por outro lado, um estudo realizado por Koenigsberg et al. (1993), utilizando-se o Therapist Verbal Intervention Inventory (TVII) e tendo por objetivo analisar as intervenções do terapeuta na psicoterapia com pacientes Borderline e monitorar o uso da técnica expressiva (esclarecimentos, confrontação e interpretação) e de apoio, encontraram como resultado uma predominância das intervenções expressivas, que variaram uniformemente de $68 \%$ a $98 \%$ entre as 12 sessões analisadas, com exceção de um caso isolado em $43 \%$. Esses resultados contrariam os encontrados no presente estudo, no qual as intervenções examinativas psicanalíticas (assinalamento e confrontação) ocuparam o terceiro lugar na classificação das mais utilizadas no tratamento, atingindo um percentual de $15,1 \%$ e, as intervenções explicativas psicanalíticas (interpretação) representaram $0,2 \%$ no processo investigado.

No entanto, mesmo que em proporções menores ao do estudo de Koenigsberg et al. (1993), no presente estudo o percentual de intervenções examinativas psicanalíticas $(15,1 \%)$, foi significativamente maior do que as explicativas psicanalíticas (0,2\%). Segundo Kernberg (2003), na psicoterapia psicanalítica com pacientes com Transtorno de Personalidade Borderline, as intervenções como assinalamento e confrontação ocupam um espaço muito mais amplo do que a interpretação.

Percebe-se que os resultados do presente trabalho estão em consonância com a literatura, que, no caso de pacientes com menos recursos, sugere a psicoterapia de apoio, ou seja, preferência por intervenções não focalizadas na transferência (Gabbard, 1984/1998). Leichsenring e Leibing (2007) colocam que as intervenções de apoio visam fortalecer habilidades que temporariamente não são acessíveis a um paciente, devido ao estresse agudo (por exemplo, eventos traumáticos) ou que não tenham sido suficientemente desenvolvidas (por exemplo, controle de impulso em Transtorno de Personalidade Borderline).

Por sua vez, analisando-se as intervenções sob o vértice das etapas do tratamento, é possível verificar que os tipos de intervenções que predominaram nas três etapas do processo coincidiram e houve uma preponderância das intervenções empáticas e exploratórias fechadas ao longo do tratamento. Assim, a terapeuta manteve os mesmos tipos de intervenções e pareceu empenhar-se para desenvolver uma aliança terapêutica entre a dupla e adotar uma postura mais diretiva.

Uma análise mais específica de cada tipo de intervenção indica que entre a primeira e a segunda etapa não houve uma oscilação significativa nas proporções de cada tipo de intervenção. 
Ao contrário, na terceira etapa, que antecede a internação psiquiátrica da paciente, houve um aumento do percentual de intervenções empáticas. Assim, observa-se a habilidade da terapeuta em sintonizar-se com a situação da paciente em crise e flexibilizar a técnica, aumentando o uso das intervenções empáticas. Essa atitude da terapeuta está de acordo com a literatura no sentido de que o terapeuta modifica suas intervenções de acordo com a sintomatologia do paciente (Goodman et al., 2014).

A análise das intervenções específicas e comuns do caso da paciente Borderline estudada indicou que $15 \%$ das intervenções utilizadas ao longo do processo investigado foram específicas psicanalíticas, enquanto que $85 \%$ foram intervenções comuns, que não fazem parte explícita do marco teórico da terapeuta e que são utilizadas em tratamentos psicanalíticos e cognitivos comportamentais. Os resultados obtidos no presente estudo coincidem com os resultados encontrados por Roussos et al. (2010), que utilizaram o nível descritivo do mesmo instrumento (CMIP-M) para examinar as intervenções utilizadas em dois tratamentos psicoterapêuticos: um cognitivo com diagnóstico de transtorno de ansiedade generalizada e um de orientação psicanalítica com diagnóstico de transtorno de personalidade não especificado e, obtiveram como resultado, que uma média de $17 \%$ das intervenções na clínica psicanalítica e uma média de $16 \%$ no tratamento cognitivo, foram específicos dos quadros teóri$\cos$.

A partir dos resultados dos estudos, percebe-se que o percentual de intervenções específicas de cada marco teórico, se mantém semelhante nos casos de transtorno de personalidade. Assim, alguns autores colocam que as psicoterapias têm se mostrado eficazes por meio de diferentes abordagens e em diferentes transtornos, sendo que tanto fatores comuns quanto fatores específicos têm sido investigados e relacionados ao sucesso terapêutico (Brum et al., 2012; Isolan et al., 2008). Autores como Roussos et al. (2010) sugerem que os fatores não específicos podem vir a ser os principais promotores de mudança em psicoterapia, e que devemos estudar fatores inespecíficos a fim de encontrar as suas ligações com os fatores específicos e, por esse viés, chegar aos fatores que promovem a mudança na psicoterapia. Outros estudiosos como Gumz, Treese, Marx, Strauss e Wendt (2015) e Pfammatter e Tschacher (2012) destacaram que a técnica e os fatores comuns não podem ser considerados independentemente. Os fatores comuns evoluem no contexto da relação terapêutica, que, por sua vez, é influenciada, em grande medida, pelas técnicas.

Segundo Roussos et al. (2010), uma possível justificativa para as diferenças encontradas nas proporções de intervenções não específicas e específicas poderia ser a de que ambos os tipos de intervenções interagem e se complementam. Considerando este pressuposto, as intervenções inespecíficas poderiam estar abrindo caminho para as específicas, isto é, sua função na psicoterapia seria a de gerar o contexto correto para as intervenções específicas. Os autores mencionam que enquanto os psicanalistas não interpretam, sinalizam ou confrontam seus pacientes, certamente não estão em completo silêncio o tempo todo. Eles utilizam intervenções inespecíficas - tais como solicitações de informação e interrogatórios fechados - para recuperar a entrada necessária ou para gerar o momento certo para realizar uma interpretação precisa. Portanto, as intervenções não específicas podem ser concebidas como "intervenções preparatórias" ou como parte do ambiente natural das intervenções específicas.

\section{Considerações Finais}

Diante da importância do aprimoramento do trabalho psicoterápico com pacientes com TPB, este estudo voltou-se para a identificação dos tipos de intervenções de um terapeuta psicanalítico na psicoterapia de uma paciente com Transtorno de Personalidade Borderline. Assim, buscou-se contribuir para a investigação da ação terapêutica no modelo psicanalítico de psicoterapia, considerando especificamente a contribuição do terapeuta no processo. Investigar as intervenções realizadas ao longo do processo terapêutico permite auxiliar tanto a pesquisa sobre as dificuldades da escolha da técnica e da moda- 
lidade de intervenções, quanto o ensino da condução da psicoterapia de orientação psicanalítica com esses pacientes.

As intervenções que sustentaram o processo investigado foram as empáticas, as exploratórias fechadas e as examinativas psicanalíticas. Nesse sentido, observa-se que o terapeuta privilegiou o desenvolvimento da aliança terapêutica e o apoio a paciente, adotando uma postura mais diretiva, ainda que utilizasse em menor frequência as intervenções psicanalíticas.

Tratando-se de um estudo descritivo, supõe-se ter aberto caminho para novas pesquisas, uma vez que o estudo apresenta limitações especialmente no que se refere à generalização dos dados. São importantes mais estudos a fim de confirmar se a escolha das intervenções utilizadas neste caso ocorreu devido às características dos pacientes com TPB ou a particularidades da terapeuta, bem como investigar fatores específicos e comuns e sua relação ao sucesso terapêutico.

\section{Referências}

Ablon, J. S., \& Jones, E. E. (1998). How expert clinicians' prototypes of an ideal treatment correlate with outcome in psychodynamic and cognitive-behavioral therapy. Psychotherapy Research, 8(1), 71-83. doi: 10.1080/10503309812331332207

Banon, E., Perry, J. C., Semeniuk, T., Bond, M., Roten, Y., Hersoug, A. G., \& Despland, J. N. (2013). Therapist interventions using the Psychodynamic Interventions Rating Scale (PIRS) in dynamic therapy, psychoanalysis and CBT. Psychotherapy Research, 23(2), 121-136. doi: 10.1080/10503307.2012.745955

Bleichmar, N. M. (1992). A psicanálise depois de Freud. Porto Alegre, RS: Artes Médicas.

Brum, E. H. M., Frizzo, G. B., Gomes, A. G., Silva, M. R., de Souza, D. D., \& Piccinini, C. A. (2012). Evolução dos modelos de pesquisa em psicoterapia. Estudos de Psicologia (Campinas), 29(2), 259-169. doi: 10.1590/S0103$-166 \times 2012000200012$

Clarkin, J. F., Fonagy, P., \& Gabbard, G. O. (2013). Psicoterapia psicodinâmica para transtorno da personalidade: Um manual clínico. Porto Alegre, RS: Artmed.
Cordioli, A. V., \& Giglio, L. (2008). Como atuam as psicoterapias: Os agentes de mudança e as principais estratégias psicoterápicas. In A. V. Cordioli (Ed.), Psicoterapias: Abordagens atuais (pp. 42-57). Porto Alegre, RS: Artmed.

Eizirik, M., \& Fonagy, P. (2009). Mentalization-based treatment for patients with borderline personality disorder: An overview. Revista Brasileira Psiquiatria, 31(1), 72-75. doi: 10.1590/S151644462009000100016

Elliot, R. (2012). Qualitative Methods for Studying Psychotherapy change Process: A guide for students and practitioners. Washington, DC: Wiley.

Fernández-Alvarez, H., Gómez, B., \& García, F. (2015). Bridging the gap between research and practice in a clinical and training network: Aigle's Program. Psychotherapy Research, 25(1), 8494. doi: 10.1080/10503307.2013.856047

Fonagy, P., \& Bateman, A. W. (2007). Mentalizing and borderline personality disorder. Journal of Mental Health, 16(1), 83-101. Retrieved from http://www.ncbi.nlm.nih.gov/pmc/articles/ PMC2816926/

Gabbard, G. O. (1998). Psiquiatria psicodinâmica: Baseado no DSM-IV (2. ed.). Porto Alegre, RS: Artes Médicas. (Original publicado em 1984)

Gabbard, G. O. (2005). Psicoterapia psicodinâmica de longo prazo: Texto básico. Porto Alegre, RS: Artmed.

Gabbard, G. O., \& Westen, D. (2003). Repensando a ação terapêutica. Revista Psiquiatria, 25(2), 257273. doi: 10.1590/S0101-81082003000200003

Goodman, G., Anderson, K., \& Diener, M. J. (2014). Processes of therapeutic change in psychodynamic therapy of two inpatients with borderline personality disorder. Journal of Psychotherapy Integration, 24(1), 30-45. doi: 10.1037/ a0035970

Goodman, G., Edwards, K., \& Chung, H. (2013). The relation between prototypical processes and psychological distress in psychodynamic therapy of five inpatients with borderline personality disorder. Clinical Psychology and Psychotherapy, 22(1), 83-95. doi: 10.1002/cpp.1875

Gumz, A., Treese, B., Marx, C., Strauss, B., \& Wendt, H. (2015). Measuring verbal psychotherapeutic techniques: A systematic review of intervention characteristics and measures. 
Frotiers in Psychology, 6(1705). doi: 10.3389/ fpsyg.2015.01705

Hallquist, M. N., \& Pilkonis, P. A. (2012). Refining the phenotype of borderline personality disorder: Diagnostic criteria and beyond. Personality Disorders: Theory, Research, and Treatment, 3(3), 228-246. doi: 10.1037/a0027953

Heinomen, E., Knekt, P., Jääskeläinen, T., \& Lindfors, O. (2014). Therapists' professional and personal characteristics as predictors of outcome in long-term psychodynamic psychotherapy and psychoanalysis. European Psychiatry, 29, 265274. Retrieved from http://www.ncbi.nlm.nih. gov/pubmed/24060629

Isolan, L., Pheula, G., \& Cordioli, A. V. (2008). Fatores comuns e mudanças em psicoterapia. In A. V. Cordioli (Ed.), Psicoterapias: Abordagens atuais (pp. 58-73). Porto Alegre, RS: Artmed.

Jordão, A. B., \& Ramires, V. R. R. (2010). Adolescência e organização de personalidade borderline: Caracterização dos vínculos afetivos Paidéia (Ribeirão Preto), 20(47), 421-430. doi: 10.1590/S0103-863X2010000300014

Kernberg, O. F. (1995). Transtornos graves de personalidade: Estratégias psicoterapêuticas. Porto Alegre, RS: Artes Médicas.

Kernberg, O. F. (2003). Psicanálise, psicoterapia psicanalítica e psicoterapia de apoio: Controvérsias contemporâneas. In A. Green (Ed.), Psicanálise contemporânea: Revista Francesa de psicanálise (pp. 23-49). Rio de Janeiro, RJ: Imago.

Kernberg, O. F., Selzer, M. A., Koenigsberg, H. W., Carr, A. C., \& Appelbaum, A. H. (1991). Psicoterapia psicodinâmica de pacientes borderline. Porto Alegre, RS: Artes Médicas.

Koenigsberg, H. W., Kernberg, O. F., Appelbaum, A. H., \& Smith, T. (1993). A method for analyzing therapist interventions in the psychotherapy of borderline patients. Journal of Psychotherapy Practice and Research, 2(2), 119-134. Retrieved from http://www.ncbi.nlm.nih.gov/pmc/articles/ PMC3330333/

Lambert, M. (2013). Bergin and Garfield's handbook of psychotherapy and behavior change. In K. A. Tompkins \& J. K. Swift (Eds.), Psychotherapy Process Outcome Research. Hoboken, NJ: John Willey \& Sons

Leichsenring, F., \& Leibing, E. (2007). Psychodynamic psychotherapy: A systematic review of techniques, indications and empirical evi- dence. Psychology and Psychotherapy: Theory, Research and Practice, 80, 217-228. doi: 10.1348/147608306X117394

Madeddu, F., Aquaro, P., \& Preti, E. (2012). Psicoterapia per il disturbo borderline di personalità: revision della letteratura sull'efficacia di quattro trattamenti manualizzati. Journal of Psychopathology, 18, 196-209. Retrieved from http://www.jpsychopathol.it/issues/2012/vol183/02Madeddu.pdf

Owen, J., Drinane, J. M., Idigo, K. C., \& Valentine, J. C. (2015). Psychotherapist effects in metaanalyses: How accurate are treatment effects? Psychotherapy, 52(3), 321-328. Retrieved from http://psycnet.apa.org/journals/pst/52/3/321.pdf

Owen, J., \& Hilsenroth, M. J. (2011). Interaction between alliance and technique in predicting patient outcome during psychodynamic psychotherapy. The Journal of Nervous and Mental Disease, 199(6), 384-389. doi: 10.1097/ NMD.0b013e31821cd28a.

Pasini, T. F., \& Dametto, J. (2010). Abordagem psicodinâmica do paciente borderline. Perspectiva, 34(128), 133-149. Recuperado em http:// pt.slideshare.net/cinthyabretas/paciente-borderline-e-seu-tratamento-pdf

Peuker, A. C., Habigzang, L. F., Koller, S. H., \& Araujo, L. B. (2009). Avaliação de processo e resultado em psicoterapias: Uma revisão. Psicologia em Estudo, 14(3), 439-445. doi: 10.1590/ S1413-73722009000300004

Pfammatter, M., \& Tschacher, W. (2012). Wirkfaktoren der Psychotherapie: eine Übersicht und Standortbestimmung. Zeitschrift für Psychiatrie Psychologie und Psychotherapie, 60, 67-76. doi 10.1024/1661-4747/a000099

Quadros, E. A., \& Yoshida, E. M. P. (2012). Variáveis do terapeuta: Análise em periódicos brasileiros (1998-2007). Interação Psicologia, 16(1), 63-72. Recuperado em http://ojs.c3sl.ufpr.br/ojs/ index.php/psicologia/article/view/11546/19716

Quagliatto, H. S. M., \& Santos, R. G. (2004). Psicoterapia psicanalítica e acompanhamento terapêutico: Uma aliança de trabalho. Psicologia Ciência e Profissão, 24(1), 74-81. doi: 10.1590/s141498932004000100009

Resolução n. 466, de 12 de dezembro de 2012. (2012). Diretrizes e normas regulamentadoras de pesquisas envolvendo seres humanos. Retrieved from http://conselho.saude.gov.br/resolucoes/2012/Reso466.pdf 
Roussos, A. J., Etchebarne, I., \& Waizmann, V. (2005). Un esquema clasificatorio para lãsintervenciones en psicoterapia cognitiva y psicoanalítica. Anuario de Investigaciones, 8, 41-48. Retrieved from http://www.scielo.org.ar/pdf/ anuinv/v13/v13a05.pdf

Roussos, A. J., Waizmann, V., \& Etchebarne, I. (2010). Common Interventions in two single cases of cognitive and psychoanalytic psychotherapies. Journal of Psychotherapy Integration, 20(3), 327-346. Retrieved from http://psycnet.apa.org/index.cfm?fa=buy. optionToBuy\&id=2010-17586-004

Tanesi, P. H. V., Yazigi, L., Fiore, M. L. M., \& Pitta, J. C. N. (2007). Adesão ao tratamento clínico no transtorno de personalidade borderline. Estudos de Psicologia (Natal), 12(1), 71-78. Retrieved from http://www.scielo.br/pdf/epsic/v12n1/ a09v12n1.pdf

Waizmann, V., Etchebarne, I., \& Roussos, A. J. (2004). La interacción entre las intervenciones psicorapéuticas de distintos marcos teóricos y los factores comunes a las psicoterapias. Revista Argentina de Clínica Psicológica, 13(3), 233-244. Retrieved from: http://www.academia. edu/3996581/La_interacci\%C3\%B3n_entre las_intervenciones_psicorap $\% \mathrm{C} 3 \% \mathrm{~A} 9 \mathrm{uticas}$ de_distintos_marcos_te $\% \mathrm{C} 3 \% \mathrm{~B} 3$ ricos_y_los factores_comunes_a_las_psicoterapias_Waizmann_Etchebarne_and_Roussos_2004_

Waizmann, V., Jurkowski, L., \& Roussos, A., J. (2006). Descripción de las intervenciones elegidas por psicoterapeutas psicoanalíticos y cognitivos. Anuario de Investigaciones, 14, 41-48. Retrieved from http://www.scielo.org.ar/pdf/ anuinv/v14/v14a04.pdf
Recebido: $23 / 09 / 2016$

$1^{a}$ revisão: $11 / 04 / 2017$

Aceite final: 30/08/2017

(c) BY (C) O(s) autor(es), 2018. Acesso aberto. Este artigo está distribuído nos termos da Licença Internacional Creative Commons Atribuição 4.0 (http://creativecommons.org/licenses/by/4.0/), que permite o uso, distribuição e reprodução sem restrições em qualquer meio, desde que você dê crédito apropriado ao(s) autor(es) original(ais) e à fonte, fornecer um link para a licença Creative Commons e indicar se as alterações foram feitas. 